Basement membrane components

\section{Basement membrane components}

\section{A C Y Li, R P H Thompson}

macrophages, lymphocytes, plasma cells, ${ }^{12}$ and mast cells. ${ }^{13}$

\section{THE GUT EPITHELIAL BASEMENT MEMBRANE}

The gut epithelial basement membrane is a specialised structure composed of ECM components. Basement membranes are ubiquitous and form stable sheets through self assembly. They support and separate the epithelium from the underlying connective tissue or lamina propria, but they also influence the behaviour of epithelial cells by controlling their shape, gene expression, adhesion, migration, proliferation, and apoptosis. ${ }^{14}{ }^{15}$ Gut basement membrane consists, as elsewhere in the body, of laminins, collagens (predominantly collagen IV), proteoglycans, calcium binding proteins such as fibulin, and various other structural or adhesive proteins. ${ }^{15}$

The major non-collagenous components are the heterotrimeric laminin glycoproteins, of which three distinct forms have been identified in human small intestinal epithelium. These are laminin $1 \quad(\alpha 1 \beta 1 \gamma 1)$ and laminin 5 $(\alpha 3, \beta 3, \gamma 2)$, which are normally expressed at the base of villus cells, and laminin $2(\alpha 2 \beta 1 \gamma 1)$, which is usually found in the crypts. ${ }^{16}$ They play a crucial role in epithelial cell anchoring, cell migration, and the differentiation of epithelial cells, and are manufactured by mesenchymal cells in the ECM. ${ }^{17}$

of fissue fibrosis and response non-surgical treatment in Crohn's disease"

At another level, ECM remodelling may be controlled by growth factors, including transforming growth factor $\beta 1$ (TGF $\beta 1$ ) and insulin-like growth factor 1 (IGF-1). ${ }^{8}$ Experiments on extraintestinal tissue show that TGF $\beta 1$ and IGF-1 both stimulate collagen deposition by fibroblasts, whereas they reduce the cellular expression of MMP. In addition, TGF $\beta 1$ increases TIMP-1 expression..$^{9-11}$

Little is known of the cells that manufacture ECM and collagen in IBD. In the damaged liver, the activated stellate cell produces ECM and collagen, and in chronic liver injury, stellate cells proliferate and mediate fibrosis. The situation is less clear in the intestine, but there may be a population of mesenchymal cells that becomes fibrogenic under the influence of cytokines or other factors. These have been classified as fibroblasts, SMC, and subepithelial myofibroblasts (SEMF). ${ }^{2}$ Fibroblasts are the major cellular component of loose connective tissue, whereas SMC are confined to the muscularis mucosa. SEMF are similar to fibroblasts, but they possess $\alpha$ smooth muscle actin. Inflammatory cell infiltrates in IBD include
BASEMENT MEMBRANE AND ECM CHANGES IN IBD

Much work has concentrated on the pathophysiology of intestinal fibrosis, especially in relation to $\mathrm{CD}$, but there are relatively few studies on the epithelial basement membrane in IBD. Changes in basement membrane structure have been identified in colonic tissue from patients with UC, with loss of immunoreactivity to laminin in the basement membrane accompanied by an increase in collagen IV and V expression. These changes paralleled the severity of inflammation within individual patients. ${ }^{18}$ Such alterations in basement membrane composition may mirror observed alterations of epithelial integrity, manifest by fluid and electrolyte loss, increased migration of bacteria, and accumulation of lymphocytes.

Mast cells reside in connective tissue, secrete numerous inflammatory mediators, and also may be implicated in intestinal fibrosis. They localise with laminin in CD strictures, and surprisingly laminin is present not only in the basement membrane of submucosal blood vessels but also deposited within the muscularis propria. This laminin teinase (MMP) enzymes, of which are at least 20, have been implicated in 
may have been derived from either the mast cells or the surrounding SMC, because mast cells were found at the centre of each deposit. It was further suggested that laminin, mast cells, and SMC interact to promote stricture formation. $^{13}$

Altered expression of the $\alpha 1, \alpha 2$, and $\alpha 3$ subunits of laminin has been noted in the inflamed segments of CD small intestine, and thus laminin 2 in intestinal crypts was replaced by laminin 1 and laminin 5. Increased expression of the $\alpha$ l subunit is of particular importance because, as opposed to $\alpha 2$ and $\alpha 3$, it is also expressed by non-epithelial cellular elements, namely SMC and blood vessels. ${ }^{16}$ It is conceivable that in inflamed tissue, SMC or blood vessels, rather than the basement membrane, are primarily responsible for the increased laminin al subunit expression. This could lead to fibrosis in the muscularis mucosa, as seen in UC and transmurally in CD.

\section{BASEMENT MEMBRANE} COMPONENTS IN SERUM IN IBD

Koutroubakis et al measured serum concentrations of two components of the basement membrane, laminin and collagen IV, in 170 patients with IBD (86 with UC and 84 with CD), and control groups consisting of 23 patients with non-IBD intestinal inflammation and 80 healthy subjects, and correlated the concentrations with the severity of the disease. ${ }^{4}$ They found that patients with both UC and CD had higher serum laminin and lower collagen IV concentrations than both non-IBD inflammatory and healthy control subjects. A weak correlation was seen between serum laminin and CRP and a negative one with albumin. None was found between collagen IV and albumin or CRP. No correlation was found between the two basement membrane components and two standard assessments of IBD activity, namely the Crohn's disease activity index and the simple clinical colitis activity index.

Interestingly, seven patients with IBD in the cohort also suffered from primary sclerosing cholangitis (PSC), which is associated with IBD, and these patients had significantly higher collagen IV concentrations than did their non-PSC counterparts. Six other patients with non-PSC liver disease were excluded. In a previous study, serum aminoterminal propeptide of type III procollagen (SPIIINP), a hepatic marker of ongoing fibrosis and inflammation, was raised in PSC. ${ }^{19}$ More recently, both laminin and S-PIIINP were found to be raised in only $19 \%$ and $40 \%$ of patients with IBD, respectively, although increases were greater in those with laboratory abnormalities of hepatic or pancreatic function. ${ }^{20}$ However, concentrations of neither marker correlated with IBD disease activity. In contrast, in another small study in 15 patients with CD embarking on surgery for their disease, concentrations of PIIINP and C terminal propeptide of collagen I were reduced compared with those of healthy controls before surgery. Both are markers of collagen synthesis, and so this was an unexpected result, ${ }^{3}$ especially because the same authors had previously shown in 29 patients with CD that collagen I degradation, as judged by increased serum concentrations of the C-terminal telopeptide of type I collagen, was increased in both active CD and patients with CD entering remission. ${ }^{21}$

Either serum laminin or collagen concentrations (or markers of their turnover) should increase in proportion to worsening IBD disease activity, in which there is considerable bowel inflammation and fibrosis. Thus, it seems that, although laminin concentrations may be modestly raised, markers of collagen turnover are not. One explanation could be that ECM or basement membrane components are retained or degraded locally.

\section{"It might have been more function- ally relevant to choose collagen $V$ in the context of gauging the activity of disease or intestinal fibrosis"}

Koutroubakis et al did attempt to exclude cases where concomitant liver dysfunction might alter serum ECM components, but it would be difficult to exclude cases where liver dysfunction had not disturbed liver blood tests. It could also be argued that any rise in serum laminin seen in active IBD could be confounded by other sources of serum laminin from inflamed or disrupted extraintestinal basement membranes.

It is also unclear why collagen IV was chosen as a marker of disease activity and intestinal tissue remodelling. It is true that collagen IV is the major collagen type found in basement membrane and raised concentrations may represent basement membrane disruption or turnover, but previous studies suggest that, although the serum concentrations of most types of collagen are raised with intestinal fibrosis, collagen $\mathrm{V}$ predominantly is affected. ${ }^{2}$ Therefore, it might have been more functionally relevant to choose collagen $\mathrm{V}$ in the context of gauging the activity of disease or intestinal fibrosis. ${ }^{22}$

In summary, basement membrane components are not yet ready to be markers of IBD activity, and much has to be learned of the importance of the ECM in IBD. The cell biology of the intestinal mesenchyme and the generation of intestinal fibrosis have not received as much attention as the hepatic stellate cell, and this could be a fruitful area for study, with the long term goal of inhibiting fibrosis and stenosis.

\section{ACKNOWLEDGEMENTS}

ACYL and RPHT receive support from Action Medical Research.

J Clin Pathol 2003;56:885-887

\section{Authors' affiliations}

A C Y Li, R P H Thompson, Gastrointestinal Laboratory, The Rayne Institute, St Thomas' Hospital, London SEl, UK

Correspondence to: Sir R Thompson, Gastrointestinal Laboratory, The Rayne Institute, St Thomas' Hospital, Lambeth Palace Rd, London SEl 7EH, UK

richard.thompson@kcl.ac.uk

\section{REFERENCES}

1 Stallmach A, Schuppan D, Riese $\mathrm{HH}$, et al. Increased collagen type III synthesis by fibroblasts isolated from strictures of patients with Crohn's disease. Gastroenterology 1992; 102:1920-9.

2 Pucilowska JB, Williams KL, Lund PK. Fibrogenesis. IV. Fibrosis and inflammatory bowe disease: cellular mediators and animal models [review] Am J Physiol Gastrointest Liver Physiol 2000:279: G653-9.

3 Kjeldsen J, Rasmussen M, Schaffalitzky de Muckadell OB, et al. Collagen metabolites in the peripheral and splanchnic circulation of patients with Crohn disease. Scand J Gastroenterol 2001;36:1193-7.

4 Koutroubakis IE, Petinaki E, Dimoulios P, et al. Serum laminin and collagen IV in inflammatory bowel disease. J Clin Pathol 2003;56:817-20.

5 Nagase H, Woessner JF, Jr. Matrix metalloproteinases. J Biol Chem 1999;274:21491-4.

6 von Lampe B, Barthel B, Coupland SE, et al. Differential expression of matrix metalloproteinases and their tissue inhibitors in colon mucosa of patients with inflammatory bowe disease. Gut 2000:47:63-73.

7 Heuschkel RB, MacDonald T, Monteleone G, et al. Imbalance of stromelysin-1 and TIMP-1 in the mucosal lesions of children with inflammatory bowel disease. Gut 2000;47:57-62.

8 Lawrance IC, Maxwell L, Doe W. Inflammation location, but not type, determines the increase in TGF-betal and IGF-1 expression and collagen deposition in IBD intestine. Inflamm Bowel Dis 2001;7:16-26.

9 Edwards DR, Murphy G, Reynolds JJ, et al. Transforming growth factor beta modulates the expression of collagenase and metalloproteinase inhibitor. EMBO J 1987:6:1899-904.

10 Gillery P, Leperre A, Maquart FX, et al. Insulinlike growth factor-I (IGF-I) stimulates protein synthesis and collagen gene expression in monolayer and lattice cultures of fibroblasts. J Cell Physiol 1992; 152:389-96.

11 Ghahary A, Shen YJ, Nedelec B, et al. Enhanced expression of mRNA for insulin-like growth factor- 1 in post-burn hypertrophic scar tissue and its fibrogenic role by dermal fibroblasts. Mol Cell Biochem 1995; 148:25-32.

12 Fiocchi C. Inflammatory bowel disease: etiology and pathogenesis. Gastroenterology 1998;115:182-205.

13 Gelbmann CM, Mestermann S, Gross V, et al. Strictures in Crohn's disease are 
characterised by an accumulation of mast cells colocalised with laminin but not with fibronectin or vitronectin. Gut 1999.45:210-17.

14 Paulsson M. Basement membrane proteins: structure, assembly, and cellular interactions. Crit Rev Biochem Mol Biol 1992;27:93-127.

15 Timpl R. Macromolecular organization of basement membranes. Curr Opin Cell Biol 1996;8:618-24.

16 Bouatrouss Y, Herring-Gillam FE, Gosselin J, et al. Altered expression of laminins in Crohn's disease small intestinal mucosa. Am J Pathol 2000; 156:45-50
17 Simon-Assmann P, Lefebvre O, BellissentWaydelich A, et al. The laminins: role in intestinal morphogenesis and differentiation. Ann N Y Acad Sci 1998:859:46-64.

18 Schmehl K, Florian S, Jacobasch G, et al. Deficiency of epithelial basement membrane laminin in ulcerative colitis affected human colonic mucosa. Int J Colorectal Dis 2000; 15:39-48.

19 Leidenius MH, Risteli LT, Risteli JP, et al. Serum aminoterminal propeptide of type III procollagen (S-PIIINP) and hepatobiliary dysfunction in patients with ulcerative colitis. Scand J Clin Lab Invest 1997;57:297-305.
20 Heikius B, Niemela O, Niemela S, et al. Elevated serum PIIINP and laminin in inflammatory bowel disease indicate hepatobiliary and pancreatic dysfunction. Hepatogastroenterology 2002;49:404-11

21 Kjeldsen J, Schaffalitzky de Muckadell OB, Junker P. Seromarkers of collagen I and III metabolism in active Crohn's disease. Relation to disease activity and response to therapy. Gut 1995;37:805-10.

22 Graham MF, Diegelmann RF, Elson CO, et al. Collagen content and types in the intestina strictures of Crohn's disease. Gastroenterology 1988;94:257-65

\section{$\mathrm{ECHO}$}

Role of microbiological investigations in the management of non-perineal cutaneous abscesses

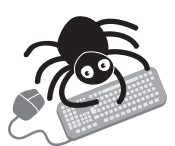

Please visit the Journal of Clinical Pathology website [www. jclinpath.com] for a link to the full text of this article.
Background: Pus samples for microbiological examination are routinely sent after incision and drainage of abscesses. There is no evidence that microbiology reports influence treatment for non-perineal cutaneous abscesses.

Aims: This study assessed (1) how often the microbiology report is used to manage patients' treatment after incision and drainage of an abscess and (2) junior surgical trainees' opinions on sending pus for microbiological examination.

Method: A retrospective analysis of the notes of all patients undergoing incision and drainage of abscesses from January 2001 to January 2002 was made. A telephone poll of junior surgical trainees was also undertaken.

Results: Most patients, $91 \%$, had specimens referred for microbiology. Of these $43 \%$ yielded no growth. Staphylococcus aureus was the most common organism (55.9\% of all positive cultures). Anaerobes were a frequent finding from axilla and groin abscesses. Mycobacterium tuberculosis was grown in two patients. Other less common organisms cultured were methicillin resistant $S$ aureus $(\mathrm{n}=1)$ and Proteus sp $(\mathrm{n}=1)$. Follow up of microbiology reports was found to be inadequate.

Conclusion: The bacteria present in non-perineal cutaneous abscesses are, for the most part, predictable. However, a significant number grow less common organisms. It is concluded that pus specimens should be sent routinely for culture and sensitivity and there should be further emphasis on following up microbiology reports by junior medical staff.

A Postgraduate Medical Journal 2003;79:519-521. 